\title{
THE GENERALIZATION OF THE PERIODIC TABLE: THE "PERIODIC TABLE" OF "DARK MATTER"
}

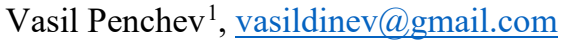

\begin{abstract}
The thesis is: the "periodic table" of "dark matter" is equivalent to the standard periodic table of the visible matter being entangled. Thus, it is to consist of all possible entangled states of the atoms of chemical elements as quantum systems. In other words, an atom of any chemical element and as a quantum system, i.e. as a wave function, should be represented as a non-orthogonal in general (i.e. entangled) subspace of the separable complex Hilbert space relevant to the system to which the atom at issue is related as a true part of it. The paper follows previous publications of mine stating that "dark matter" and "dark energy" are projections of arbitrarily entangled states on the cognitive "screen" of Einstein's "Mach's principle" in general relativity postulating that gravitational field can be generated only by mass or energy.
\end{abstract}

Key words: dark matter, dark energy, entanglement, Periodic table, separable complex Hilbert space

\section{SYNOPSIS}

The paper follows a previous a presentation in Torino (2019 July 15) also published as a paper (Penchev 2019). It discusses a thought experiment in Einstein's manner of "Gedankenexperimenten" to suggest a possible generalization of the invariance of physical laws to arbitrarily accelerated reference frames in general relativity in relation to chemistry.

The conclusion claims the indistinguishability of the following two kinds of the spectral lines of the same chemical substance: (1) due to red shift and originating from an arbitrarily remote astronomical object (such as a star, a galaxy, etc.), anywhere in the universe; and (2), after entanglement in experiments conducted on our planet; furthermore (3), a bijection exists between all possible red shifts and those shifts due to entanglement.

In other words, as accelerated motion and gravity are indistinguishable after Einstein's Gedankenexperiment in general relativity, as accelerated motion, gravity, and entanglement are indistinguishable under the conditions of the thought experiment suggested in the previous paper and presentation.

The conclusion relies on an eventual conservation of quantum information (considered as the Noether correlate of the physical quantity of action) and generalizing energy and matter conservation in physics and chemistry. Particularly, a generalized Periodic table of entangled chemical elements can be put forward as identical to standard one but dispersed in any possible motion anywhere in the universe and accessible by spectral observations on our planet.

The article accepts the existence of "dark matter" (Trimble 1987) and "dark energy (Riess et al. 1998) as very well established experimentally and will state that the aforementioned Periodic table of entangled chemical elements can be interpreted furthermore as the "Periodic table" of "dark matter" under a few additional conditions, which will be discussed as well. Or in other words: dark matter is due to entanglement since the pair of dark matter and dark

\footnotetext{
${ }^{1}$ Bulgarian Academy of Sciences: Institute of Philosophy and Sociology: Dept. of Philosophy of Science
} 
energy is equivalent to entanglement in virtue of quantum information conservation ${ }^{2}$; once again: dark matter is "visible" only as entangled states of the standard matter (meant e.g. by the Standard model or the Periodic table); and entanglement propagating "instantly" by quantum correlations is what adds dark matter and dark energy to the visible ones being just "visible", i.e. after propagating of "light" or any electromagnetic radiation therefore limited of the corresponding fundamental constant of light speed. This means that mass and energy can be divided disjunctively into visible or dark by the velocity of propagating interaction eider subluminal or superluminal respectively. Dark matter and dark energy available in advance are to be added to the visible ones when and if the relevant entities be observed.

Entanglement in turn is to be identified with gravity just as gravity is identified with relative acceleration in virtue of general relativity. Quantum information conservation admits the violation of energy conservation since the later should be restricted only to the subluminal area unlike quantum correlations. Then, quantum correlations meant by quantum information conservation are "depicted on the screen" of visible matter and energy (e.g. in virtue of Einstein's (1918) "Mach's principle") as invisible, or "dark" as far as they are out of that "screen" by themselves and only projected on it (metaphorically, like "shadows on the wall" of Plato's “cave").

Einstein's "Mach's principle" was introduced to justify the "cosmological constant" in the Einstein field equation. It states that only mass and energy (both visible on the temporal "screen") can be the source of gravity in the framework of general relativity being exceptionally well confirmed experimentally. Then, if any other source of gravity exists (as this follows from the option of energy non-conservation within quantum-information conservation), it would project as dark matter and dark energy in virtue of "Mach's principle" and the corresponding physical quantity of action. If that is the case, dark matter and dark energy would be observable only after gravitational phenomena and described by general relativity: just as it is in fact. No experiment in the framework of the Standard model can establish any effect of dark matter or dark energy; explicably, after it is a theory only of the visible matter and energy in definition.

The sketched solution about the "dark" problem in physics suggests the generalization of energy conservation (e.g. as quantum information conservation here), in the framework of which violations of energy conservation be admissible as not contradicting the natural laws.

Anyway, dark matter and dark energy might be interpreted "conservatively", i.e. in the framework of energy conservation therefore excluding any violations of it. As neutrino was discovered initially theoretically in virtue of energy conservation, so that any observed violation would mean the existence of yet unknown entity, but visible and confirmable by relevant experiments, some researches grant that the ostensible "darkness" is not more than an unrevealed yet "visibility" obeying the absolutely universal energy conservation.

Then the dilemma between the former and latter approach seems to be: a new law in cognition versus new entities in nature. Is the former more revolutionary? Presumably yes,

\footnotetext{
2 The eventual generalizing law of quantum information as well as its inference and proof are suggested in another previous paper (Penchev 2020 August 17), and the link of that law to the problem about dark matter and dark energy, in: Penchev 2020 August 31.
} 
as far as it implies the reformation of many scientific areas; e.g. that of chemistry discussed here:

I mean the "problem about the direct transformation of chemical substances" considered in a previous paper (Penchev 2020 June 15) as well. Indeed, though equivalent as to spectral lines, a chemical substance on a remote star only observable terrestrially and the same substance in an entangled state equivalent as to spectral lines keep to be fundamentally different as to their empirical or experimental properties. The substance being within the remote star cannot behavior like the substance which it only depicts on Earth by identical spectral lines.

On the contrary, one can conject naturally that the same substance being entangled is to behavior just as the substance only "depicted" in the former case. Consequently, if any substance is entangled so that its spectral lines turn out to be those of gold, for example, it would be indistinguishable from gold by itself.

This means: entanglement and gravity influence the final terrestrial observation differently. The former changes the substance itself; the latter, only the signal of it rather than itself. Then, the generalized "periodic table" of "dark matter" should be related to the former rather than to latter.

\section{ENTANGLEMENT ON THE SCREEN OF “MACH'S PRINCIPLE”: DARK MATTER \& DARK ENERGY, AND THEIR DIFFERENCE \\ Einstein's "Gedankenexperiment" about an accelerating elevator can be summarized as} follows in the present context. Einstein demonstrated his generalized principle of relativity by the indistinguishability of experience in the following two cases: (1) observations in an arbitrary accelerated elevator; (2) observations in the same elevator situated in a gravitational field. Thus, he inferred the mathematical and physical essence of gravitational field as a relation of two arbitrarily accelerated reference frames: what is the general principle of relativity (covariance).

The idea of indistinguishability can be generalized to the following two groups of experiments as to gravitational field:

(1) The red shift of spectral lines of a certain chemical substance in any point of the universe and observed from Earth.

(2) The red shift of spectral lines of the same substance being on Earth, but entangled relevantly for the red shift at issue.

The wave function of that chemical substance observed on Earth is modified by: (1.1) the geodesic line from any point in the universe to Earth in pseudo-Riemannian space for the gravitational field of the universe in the former case, (1.2) a second wave function entangled with that of the substance in the latter case.

Einstein's "Gedankenexperiment" demonstrates the experimental indistinguishability of gravitational field and any relevant force field causing the same acceleration. Then, the suggested thought experiment is to show an analogical, but generalized experimental indistinguishability of gravitational field to a relevant state of entanglement causing the same observed red shift. One would be to generalize the general principle of relativity (GPR) further more; namely, that the essence of gravitational field is representable by two 
equivalent ways: (1) as a relation of two arbitrarily accelerated reference frames; but (2), as a relation of two arbitrarily entangled wave functions.

That generalization of GPR postulates the equivalence of GPR and a fundamental class of quantum phenomena: those of entanglement. Thus, that generalization works implicitly as a theory of quantum gravity (though nonstandard due to the complementarity of the quantization of gravitational field unlike that of each the three interactions of the Standard model). General relativity understood as nonstandard theory of quantum gravity would state that it is a complementary, smooth description of entanglement, but equivalent to it after a relevant transformation. That relevant transformation can be described coining a new concept: "discrete (or external, or quantum) reference frame", therefore generalizing GPR from accelerated smoothly reference frames, to discrete ones (i.e. non-continuous and thus, non-smooth).

In fact, the concept of discrete reference frame as a further generalization of the concept of "reference frame" needs a generalization of time as well: from the irreversible (though arbitrarily "curved") time of general relativity to the reversible time of coherent quantum state. This implies further generalization of energy and matter (physically, a particular case of energy due to Einstein's " $E=m c^{2}$ "): namely that of dark matter \& dark energy describable whether as "atemporal" or as relevant to the reversible time of coherent state.

Indeed, that generalization is to be linked to "dark matter" and "dark energy" directly and explicitly: they can be explicated as a projection of that generalization on the "cognitive screen" of general relativity after "Mach's principle".

Einstein introduced "Mach's principle" only in 1918 to infer from it for the universe to be static adding the "cosmological constant" to the initial equation(s) of general relativity. However, "Mach's principle" is much more general stating that the "only source of gravitational field are matter and energy". In fact, "Mach's principle" is implicit even in the initial "Einstein field equation" for its structure: a space-time tensor corresponding to mass to be equated to an energy-momenta tensor for energy. So, if anything else generates gravitational field, it would imply relevant mass and energy corrections in the Einstein field equation.

Both dark matter and dark energy can be established only after astronomical observations therefore based only on general relativity and thus, "switching on the cognitive screen of Mach's principle". Dark matter is necessary for the Milky Way and many other galaxies are rotated too fast and should disintegrate and scatter due to centrifugal force since their visible mass and energy including the dark holes within them is extremely insufficient to balance it. Dark energy explains the observed acceleration of the expansion of the universe: the universe seems not to be a closed system, but obtains permanently energy, namely "dark energy" which accelerates its expansion.

Though the dark (matter \& energy) in total are about 20 times more than the visible ones, they can be considered mathematically as corresponding corrections in the Einstein field equation due to, and on the screen of "Mach's principle". Also, they can be contained implicitly in a generalized "cosmological constant" turning out to be a "cosmological function" properly. Meaning some hypothetical source only projectable on Mach's screen as 
dark matter and dark energy, it is identifiable as entanglement for the "still more general principle of relativity" demonstrated by the thought experiment in Einstein's manner in the beginning.

Dark matter is to be a "correction" in the space-time tensor, and dark energy, in that of energy-momenta correspondingly. Meaning them explicitly as being due to entanglement, they can be separated in a "cosmological function" so that its variables would be two entangled wave functions:

The one of them would correspond to the distribution of dark energy in space-time, and the other one, to the "gravitational field of dark mass" correspondingly. The usual Einstein field equation would be a particular case of the generalized one if (1) both "fields" are zero; or (2): they are orthogonal (or complimentary as in the "classical" quantum mechanics) to each other, and thus their entanglement is zero.

The Einstein field equation can be represented conceptually so:

$$
\left\{R_{\mu \nu}=\frac{8 \pi G}{c^{4}} T_{\mu \nu}\right\}+\left\{\frac{R}{2} \boldsymbol{g}_{\mu \nu}\right\}
$$

Here $R_{\mu \nu}$ is the Ricci curvature tensor; $G$ is the gravitational constant; c is the constant of light speed in a vacuum; $\mathrm{R}$ is the scalar curvature; $g_{\mu \nu}$ is the metric tensor; and $T_{\mu \nu}$ is the energy - momentum tensor.

The sense of the Einstein field equation is the following:

$\{$ The gravitational field due to mass $=$ that due to energy $\}+\{$ a function due to the metric of pseudo-Riemannian space\}. The former brackets surround the screen of "Mach's principle" properly though represented as an equation, and the latter brackets, what is to be "painted" on that screen as both dark matter and dark energy correspondingly.

Einstein introduced the "cosmological constant" in the equation in 1918:

$$
\left\{R_{\mu \nu}=\frac{8 \pi G}{c^{4}} T_{\mu \nu}\right\}+\left\{\left(\frac{R}{2}-\Lambda\right) g_{\mu \nu}\right\}
$$

The sense of adding the "cosmological constant" $\boldsymbol{\Lambda}$ is: there exists some unknown (today, "dark") influence on the relation of the gravitational field due to mass (today, "visible mass") and the gravitational field due to energy (today, "visible energy"). Furthermore, that ("dark") influence changes the metric of pseudo-Riemannian space.

Then, the cosmological constant can be generalized to a "cosmological function":

$$
\left\{R_{\mu \nu}=\frac{8 \pi G}{c^{4}} T_{\mu \nu}\right\}+\left\{\left(\frac{R}{2}-\Lambda(x, y, z, t)\right) g_{\mu \nu}\right\}
$$

The sense of generalizing the "cosmological constant" $\boldsymbol{\Lambda}$ to the "cosmological function" $\boldsymbol{\Lambda}(\boldsymbol{x}, \boldsymbol{y}, \boldsymbol{z}, \boldsymbol{t})$ is that "dark influence" is not homogeneous, constant, but different in any space-time point (in general). In other words, the transformation of pseudo-Riemannian space is accomplished by an arbitrary operator, the action of which is experimentally observable as the additional "dark matter" and "dark energy". 
At last, the cosmological function can be interpreted as two entangled quantum fields, i.e. as the "cosmological function of entanglement":

$$
\left\{R_{\mu \nu}=\frac{8 \pi G}{c^{4}} T_{\mu \nu}\right\}+\left\{\left(\frac{R}{2}-\Lambda\left[\Psi(x, y, z, t), \boldsymbol{\Theta}\left(p_{x}, p_{y}, p_{z}, E\right)\right]\right) g_{\mu \nu}\right\}
$$

That is: $\boldsymbol{\Lambda}\left[\boldsymbol{\Psi}(x, y, z, t), \boldsymbol{\Theta}\left(p_{x}, p_{y}, p_{z}, E\right)\right]$ is the "function pf entanglement" of two quantum fields:

$\boldsymbol{\Psi}(x, y, z, t)$, and $\boldsymbol{\Theta}\left(p_{x}, p_{y}, p_{z}, E\right)$. The sense of the interpretation of the cosmological function

the "cosmological function of entanglement" is: the observable dark influence is due to entanglement; that is the macroscopic total effect of the microscopic quantum entanglement. The "cosmological function of entanglement" can be projected on the "screen of mass and energy":

The structure of the Einstein field equation:

" $\{\mathrm{GF}$ due to mass $=\mathrm{GF}$ due to energy $\}+$ "dark GF" implies:

$\{\mathrm{GF}$ due to mass visible $+\mathbf{d a r k}=\mathrm{GF}$ due to energy visible + dark $\}$

\section{Dark gravitational field GF = that of dark matter (mass) + that of dark energy}

The hypothesis (a little above) was: the dark GF is due to entanglement or even, only to entanglement. Entanglement by itself is only information, therefor invisible or "dark": it seems as both dark matter \& dark energy only on the "screen" of the Einstein field equation (respectively, "Mach's principle").

After Einstein's " $E=m c^{2}$ ", energy \& matter are the same, but what? What is "the same" of matter \& energy at issue?

The advocated thesis implies that it should be temporality; in other words, "Mach's principle" can be paraphrased equivalently: "any physical process is temporal" (being equivalent to "only matter and energy are the sources of GF").

This is not valid as to entanglement: it is a physical process, but not in time since its time to occur is zero definitively, or being another source of GF, So, the "screen of Mach's principle", the "screen of the Einstein field equation", and the "screen of temporality" are the same after: "all physical is energy", and energy implies time according to the first Noether (1918) theorem.

One would question: "What about quantum mechanics and the "temporal screen" there?"

All physical quantities in quantum mechanics are Hermitian operators being consistent to the unitarity of the separable complex Hilbert space and to their general commutativity with the Hamiltonian of the system (being the operator of energy). So, if (or though) time is the only physical quantity not being an operator ${ }^{3}$, but being the conjugate of energy, it is

${ }^{3}$ That idea was suggested by Wolfgang Pauli in his debate with Niels Bohr about the "conservation of energy conservation" in quantum mechanics. Indeed, if time were an operator as all the rest 
universal not less than energy and valid to any quantity, which is a Hermitian operator. However, entanglement is equivalent to non-Hermitian operators: furthermore, out of temporal screen due to the above consideration.

Quantum information is measured in units of qubits (where "qubit" is an orthonormal superposition of any two orthogonal subspaces of the Hilbert space of quantum mechanics). Quantum information is able to unify and describe uniformly Hermitian operators (i.e. all temporal physical quantities) and non-Hermitian operators (i.e. entanglement). Consequently, quantum information is to act physically and be equivalent to a certain quantity of action, e.g. that of the Planck constant.

Since quantum information can be represented as equivalent to the information of infinite series or sets, a qubit is an infinite set of bits, and the physical action of any finite sets of classical bits is zero (as it is accepted commonly).

So, entanglement being by itself quantum information equivalent to physical action is, nonetheless, out of time. Thus, there exists physical action which is out of time and conditioning $95-96 \%$ of the total mass and energy of the universe being expressed in units of mass or energy (i.e. as if "within time").

The conclusion is: our scientific worldview is extremely incomplete and even wrong partly for ignoring all physical out of time. One may coin two metaphors of the "temporal screen": (1) as the "wall of Plato's cave"; (2) as the "screen of the quantum computer of the universe".

\section{MATER AND DARK MATTER IN CHEMISTRY AND QUANTUM CHEMISTRY: THE PERIODIC TABLE}

The proved existence of dark matter restricts all chemical knowledge (or any other until now) only within the scope of visible matter. The advocated hypothesis considers dark matter by itself as the substance of quantum information due to entanglement. Visible matter refers only to the particular case of quantum information on the "screen of time", on which it is distinguishable from visible energy.

Can the existent chemistry of visible matter be generalized as to an eventual chemistry of quantum information?

Though visible, plasma does not possess chemical properties in the usual meaning: so, chemical properties are defined standardly as to the low-energetic states not being in plasma state. They refer to the electron shell of the atom divided onto energy levels (or layers and sublayers) and especially, to the top energy level of valence electrons. All Atoms can be separated in classes according their chemical properties of about 118 chemical elements (discovered or synthesized until now). Their chemical properties are altered discretely and correspond to the number of electrons in the shell constituting periodic groups of similar chemical properties because all electron shells obey rigorous quantitative laws of quantum physics.

physical quantities in quantum mechanics, this would imply its variability after measurement and thus, a different value of energy in any single measurement, i.e. the violation of energy conservation in the final analysis. 
All chemical elements can be visualized by the periodic groups of their chemical properties (i.e. of their electron shells) in a compact two-dimensional ordering famous as the "Periodic table". Meaning it, one can question about an eventual generalization or complementation of the Periodic table as to dark matter. Furthermore meaning the advocated hypothesis of dark matter as entangled states, that Periodic table of dark matter is interpreted as the meaning or relation of the Periodic table to entangled states.

A few fundamental or conceptual obstacles can be enumerated during that pathway:

The Periodic table of visible matter is discrete, but entanglement is continuous even smooth. All chemical properties can be defined (at least until now) only on the "screen of time" therefore being meaningless "out of time" unlike entanglement or quantum information. Thus, the concept of chemical property suggests the distinction of matter and energy (withal it should be the low energy of non-plasma). On the contrary, entanglement implies their indistinguishability and smooth transition (obeying an eventual generalizing conservation of quantum information, but violating energy conservation sometimes).

However, if one manages to overcome those obstacles, a new perspective to the Periodic table of visible matter would be open:

Now, after dark matter, the Periodic table can be understood as a two-dimensional series of atomic states whether stable or radioactive, but only on the "temporal screen" (or that of energy, resp. matter conservation). One can figure quantum information as a mathematical function definable as by the variable of time (the case of the "classical" quantum mechanics) as independently of it (studying the phenomena of entanglement). Then, the chemical elements as all visible matter would be the "roots ("zeros") of the equation of quantum information" to the variable of time (i.e. the points in which the "function graphic of quantum information crosses the axis of time", and time makes sense). All visible matter consists of similar "solutions" of the "equation of quantum information".

The Periodic table was invented by the Russian scientist Mendeleev by generalizing the empirical experience of chemistry, but without any reason why the Periodic law exists. Only quantum chemistry after the Bohr model of the atom managed to reduce the Periodic law to quantum properties of the corresponding electron shell, its discrete energy levels and their filling by electrons following the principle of Pauli. Regardless of wave-particle duality in quantum mechanics, as the contemporary model of the atom as the quantum foundation of chemistry prefers the language of particles rather than the equivalent language of waves.

On the contrary, the theory of quantum information and entanglement uses the "wave language". So, the appearing quantum-information chemistry of entangled chemical substances needs wave-particle duality to synthesize quantum chemistry with quantum information. For example, the electron shell of the atom of a certain chemical element is to be represented by a single wave function to be able to be investigated its entanglement with any other wave function. The reverse approach, the translation of entanglement into the language of particles, is not less admissible, but seems to be more difficult technically as well as generating inconsistence with the Standard model.

The few principles of the translation of "particle language" into "wave language" are based on "quantum number" granting it to any quantum physical quantity whether continuous 
(smooth) or discrete (properly quantum). Any physical quantity is described by a relevant Hilbert subspace: finitely dimensional for discrete quantities, but infinitely dimensional for continuous ones. Any quantum entity is described by a finite set of (even only a few) quantities therefore by that separable complex Hilbert space consisting of the same set of subspaces though some of them being infinitely dimensional. The shell properties (quantum numbers) relevant to chemistry refer only to finite Hilbert subspaces.

The "conservation of energy conservation" in quantum mechanics is a postulate imposed initially by Pauli debating against Bohr ${ }^{4}$, and now, as commonly accepted as underlying the Standard model and the quantum language of particles. If the separable complex Hilbert space unifying Heisenberg's matrix mechanics and Schrödinger's wave ("ondulatory") mechanics is granted, its property of unitarity implies energy conservation as a necessary physical interpretation of itself.

It can be justified physically very simply: if the wave function of an entity is the same to any possible apparatus, and the dual Hilbert spaces are idempotent, the former can be interpreted equivalently (and thus inferring energy conservation) so: any possible wave function of the same entity is the same (e.g. to a certain apparatus).

However, the advocated interpretation of "darkness" as entanglement needs a relevant generalized conservation therefore violating energy conservation sometimes (and even very often). That is "quantum information conservation" meaning that the set of quantum numbers is predetermined and unchangeable; furthermore: only namesake quantum numbers of two or more wave functions can be entangled; the violation of energy conservation consists in the direct transformation of quantum information into energy, but only under the condition of quantum information conservation (therefore being the source of dark matter or dark energy, and out of "Mach's principle").

Though energy and matter are equivalent after " $E=m c^{2}$ ", matter in chemistry is meant only as "matter at rest" (having mass at rest) and being non-plasma (for electron shell to exist) So, one needs the Ricci tensor of the Einstein field equation and relevant to the gravitational field of matter at rest to be represented by the entanglement of two (or more) wave functions. This is very complicated technically rather than conceptually. The Ricci tensor at issue is the Ricci tensor of the one of the two entangled quantum fields; namely: $\boldsymbol{\Psi}(x, y, z, t)$ in relation to $\boldsymbol{\Lambda}\left[\boldsymbol{\Psi}(x, y, z, t), \Theta\left(p_{x}, p_{y}, p_{z}, E\right)\right]$.

This means:

$$
R_{\mu \nu}=\left\{R\left(\Psi(\mathrm{x}, \mathrm{y}, \mathrm{z}, \mathrm{t}), \Lambda\left[\Psi(\mathrm{x}, \mathrm{y}, \mathrm{z}, \mathrm{t}), \Theta\left(\mathrm{p}_{\mathrm{x}}, \mathrm{p}_{\mathrm{y}}, \mathrm{p}_{\mathrm{z}}, \mathrm{E}\right)\right]\right) g_{\mu \nu}\right\}
$$

The result is inferred from a few previous statements ${ }^{5}$.

\footnotetext{
${ }^{4}$ The so-called BKS theory is meant (Bohr, Kramers, Slater 1924). The book (Hendry 1984) contains a detailed description of the dialogue of Bohr and Pauli including in relation of energy conservation in quantum mechanics and the BKS theory.

5 "The Einstein field equation with the "cosmological function of entanglement": $\left\{R_{\mu \nu}=\frac{8 \pi G}{c^{4}} T_{\mu \nu}\right\}+$ $\left\{\left(\frac{R}{2}-\boldsymbol{\Lambda}\left[\boldsymbol{\Psi}(x, y, z, t), \Theta\left(p_{x}, p_{y}, p_{z}, E\right)\right]\right) g_{\mu v}\right\}$. Here $\boldsymbol{\Lambda}\left[\boldsymbol{\Psi}(x, y, z, t), \Theta\left(p_{x}, p_{y}, p_{z}, E\right)\right]$ is the "function of entanglement" of two quantum fields: $\boldsymbol{\Psi}(x, y, z, t)$ and $\Theta\left(p_{x}, p_{y}, p_{z}, E\right)$. "The Ricci tensor at issue is the
} 
Quantum information chemistry is to be defined as that quantum chemistry studying the influence of entanglement. As far as the chemical properties are determined by electron shell, quantum information chemistry investigates entangled electron shells (since only namesake quantum numbers can be entangled according to quantum information conservation). Furthermore, entanglement adds "dark matter" to the visible matter of chemical substance obeying the Periodic law. So, entanglement (or respectively "dark matter") would influence or modify the Periodic table of visible matter.

The Periodic law follows the successive filling of the admissible discrete energy levels of electron shell one by one and corresponding to the serial number of chemical elements. The second dimension of the Periodic table corresponds to the number of filled energy layers (or sublayers) of electron shell. Chemical element in entangled state can remain the same, but energetically excited therefore radiating photons after decoherence. Furthermore, the entangled chemical element may be changed into another substance (not necessary element) due to the chemical properties of the entangled system.

A few problems arise immediately about the entangled chemical compound:

Can the Pauling (1960) chemical bond be interpreted as an entangled state of electrons belonging to different atoms?

Can "chemical compound" be generalized as to the entanglement (chemical bond) of nonvalence electrons of the shell?

Can entanglement generate new chemical compounds, which cannot be a result of any classical chemical reaction?

Can entanglement explain the phenomena of catalyze?

The list of problems in front of quantum-information chemistry would be too long.

One can generalize entangled electron shells by adding the entanglement of the corresponding nuclei (after "one shell implies one nucleus"). This means to be investigated the influence of entanglement of the corresponding discrete quantum numbers of atomic nuclei. Analogically to the entanglement of electron shells, the entangled atomic nucleus can remain the same, but energetically excited, and can change in another isotope or chemical element. Is nuclear fusion (needing additional energy usually) can be explained or generalized by entanglement (as "chemical compound")?

The polar consideration is possible: can any radioactive isotope be interpreted as an entangled state of the products of its decay? If yes, which is the reason for the process of decoherence after many radioactive isotopes to be so slow? The successful solution of the above problem is the key to creating huge, super-powerful and mass available quantum computers. Can one influence the speed of radioactive decay by entanglement? For example, can the chemical element "118", Oganesson with the semi-decay of 700 microseconds for ${ }^{294} \mathrm{Og}$ to be stabilized in any degree by entanglement?

Ricci tensor of the one of the two entangled quantum fields, namely $\boldsymbol{\Psi}(x, y, z, t)$ in relation to $\Lambda\left[\Psi(x, y, z, t), \Theta\left(p_{x^{\prime}}, p_{y}, p_{z}, E\right)\right] . "$ 
IV INSTEAD OF CONCLUSION: THE PERIODIC TABLE OF DARK MATTER IN TERMS OF QUANTUM INFORMATION

The only quantity which can be assigned to dark matter (as well as to dark energy) directly is quantum information, furthermore shared with visible matter and energy. All other quantities are the influence and change of the namesake quantities (quantum numbers) of visible matter and energy. One can speak of dark matter and dark energy only in that sense and continuing the list of dark quantities as corresponding counterparts of the visible ones.

If one accepts the Periodic table only as a list of quantum numbers relevant to electron shell, it will be valid to matter as visible as dark.

The influence of quantum information can be visualized as if decomposing the relevant non-Hermitian operators into pairs of Hermitian one changing a certain quantum quantity and Hamiltonian changing energy accordingly. Entanglement can be interpreted also as an omnipresent physical interaction therefore implying for any physical system not to be closed and thus changing energy permanently. Thus, entanglement is able to change the value of any quantum number (including those relevant to the Periodic table), but not to create new quantum numbers, being forbidden by quantum information conservation.

One can use the metaphor of Plato's "cave": the people are chained and thus can observe only the wall and shadows on it, but not what causes the shadows. So, the shadows can be described only in terms of the wall though those terms are irrelevant to the shadows by themselves. All possible experience of humankind is temporal: thus, the "screen of time" is what that metaphor means as depicted by the "wall of the cave" in the metaphor. On the contrary, what causes the shadows is not temporal, nonetheless being visible only as shadows of temporal screen. So, the "shadows of dark matter" can be observe only on the "screen" of the usual Periodic table of visible matter.

Any scientific hypothesis is to offer new predictions not inferable from all the rest without it (i.e. to be falsifiable in a Popper sense if predictions are not observed or even being rejected). The basic class of predictions due to the advocated hypothesis can be defined by the following option:

Energy be able to be transformed directly into quantum numbers relevant to a certain chemical substances by entanglement with another substance sharing the same quantum numbers as those undergone to change.

Anyway, one can question about the dark "Periodic table" by itself (i.e. not as a projection onto the visible Periodic table). What becomes visible on the "screen of time" (i.e. the nonHermitian operators) can be liken as incomplete quantum calculations. The complete calculations of the universe as a quantum computer are all Hermitian operators and thus physical quantities, only to which the concept of the Periodic table makes sense; rr in other words, the dark Periodic table by itself is neither "periodic" nor "table". 


\section{References:}

Bohr, N., H. A. Kramers, J. A. Slater (1924) "The quantum theory of radiation," Philosophical Magazine and Journal of Science 47 (281): 785 - 802

Einstein, A. (1918) "Prinzipielles zur allgemeinen Relativitätstheorie," Annalen der Physik 55 (4): $241-244$.

Hendry, J. (1984) The creation of quantum mechanics and the Bohr-Pauli dialogue. (Studies in the history of modern science; v. 14). Dordrecht - Boston - Lancaster: D. Reidel.

Noether, E. (1918) "Invariante Variationsprobleme," Nachrichten von der Gesellschaft der Wissenschaften zu Göttingen. Mathematisch-Physikalische Klasse 1918: 235-257.

Pauling, L. (1960) The nature of the chemical bond and the structure of molecules and crystals: an introduction to modern structural chemistry ( $3^{\text {rd }}$ edition). Ithaca, New York: Cornell University Press; London: Oxford University Press.

Penchev, V. (2019) "From the Principle of Least Action to the Conservation of Quantum Information in Chemistry: Can One Generalize the Periodic Table?" Chemistry: Bulgarian Journal of Science Education 28 (4): 525-539 (SSRN: https://ssrn.com/abstract=3644084 ); presentation at Torino, 2019 July 15: https://easychair.org/smart-slide/slide/1KV7\#

Penchev, V. (2020 June 15) "Problem of the Direct Quantum-Information Transformation of Chemical Substance," SSRN: https://ssrn.com/abstract=3627003 or http://dx.doi.org/10.2139/ssrn.3627003

Penchev, V. (2020 August 17) "Quantum-Information Conservation. The Problem About 'Hidden Variables', or the 'Conservation of Energy Conservation' in Quantum Mechanics: A Historical Lesson for Future Discoveries," SSRN: https://ssrn.com/abstract=3675319 or http://dx.doi.org/10.2139/ssrn.3675319

Penchev, V. (2020 August 31) "Two deductions: (1) from the totality to quantum information conservation; (2) from the latter to dark matter and dark energy, 2020)," SSRN: https://ssrn.com/abstract=3683658 or http://dx.doi.org/10.2139/ssrn.3683658

Riess, A. et al. (Supernova Search Team) (1998) "Observational evidence from supernovae for an accelerating universe and a cosmological constant," Astronomical Journal 116 (3): 1009-1038.

Trimble, V. (1987) "Existence and nature of dark matter in the universe," Annual Review of Astronomy and Astrophysics 25 (1): 425-472. 